Erneut ist knapp ein Jahr vergangen, und für die Herausgeber ist es Anlaß zur Freude, den vierten Band des Jahrbuchs vorlegen zu können. Neben den bewährten Landesberichten über die Schweiz und die Vereinigten Staaten (3.a) enthält er die Fortsetzung der Abhandlung zu Berlin (3.b); Schwerpunkt der merklich erweiterten Dokumentation ist die italienische Referendumspraxis (2.b). Die Beiträge im Hauptteil widmen sich dem Verhältnis von Direkter Demokratie und Frauenstimmrecht, der Analyse der langwelligen Entwicklung in den deutschen Bundesländern sowie anhand eines bayerischen Bürgerentscheides einer neuartigen politikwissenschaftlichen Analysemethode (1.). Im Rechtsprechungsteil ist schließlich die durchaus überraschende Entscheidung des Bayerischen Verfassungsgerichtshof in Sachen Studienbeiträgen zu verzeichnen (7.).

Der Dank der Herausgeber gilt einmal mehr dem Vorstand von „Mehr Demokratie“ e.V. sowie dem Nomos Verlag für die Unterstützung und umsichtige Betreuung des Jahrbuchs. Zu danken haben wir ferner den Mitarbeiterinnen und Mitarbeitern der Münsteraner Professur für Öffentliches Recht, Rechtsphilosophie und Rechtssoziologie für ihre Mitwirkung an der Recherche sowie der Korrektur der Manuskripte und Fahnen. Besonders erwähnt seien in diesem Jahr einmal mehr Herr cand. iur. Marcel Wirth, ferner Frau stud. iur. Nina Mruk, Herr stud. iur. Lutz Friedrich sowie die Wissenschaftlichen Mitarbeiter Nana K. A. Baidoo und Martin Minkner. 
Inhaltsverzeichnis

Vorwort

1. Abhandlungen

Nadja Braun Binder/Hans-Urs Wili-Luginbühl

„Die ersten werden die letzten sein“ und „Die Frau soll in der Versammlung schweigen“. Direkte Demokratie und Frauenstimmrecht

Fabian Wittreck

Direkte Demokratie in den Ländern - Kontingenter Prozeß oder List der Vernunft?

Marcel Preising/Martin Messingschlager

Zur Erklärung und Prognose des Abstimmungsverhaltens bei Bürgerentscheiden: Das Beispiel einer Kommune in der fränkischen Provinz

Otmar Jung

Dian Schefold zur (direkten) Demokratie

2. Dokumentation

3. Landesberichte

a) Internationale Ebene

Axel Tschentscher/Dominika Blonski/Rahel Baumgartner

Direkte Demokratie in der Schweiz - Landesbericht 2012 
Hermann K. Heußner

Direkte Demokratie in den US-Gliedstaaten in den Jahren 2010 und 2011

- Ein Überblick

b) Deutschland

\section{Otmar Jung}

Die Reform der direkten Demokratie in Berlin 2006 (Teil II)

4. Rechtsprechung

Fabian Wittreck

Ausgewählte Entscheidungen zur direkten Demokratie

5. Rezensionsabhandlungen

6. Neue Literatur

Die Herausgeber

Über die Autorinnen und Autoren

Sachverzeichnis 